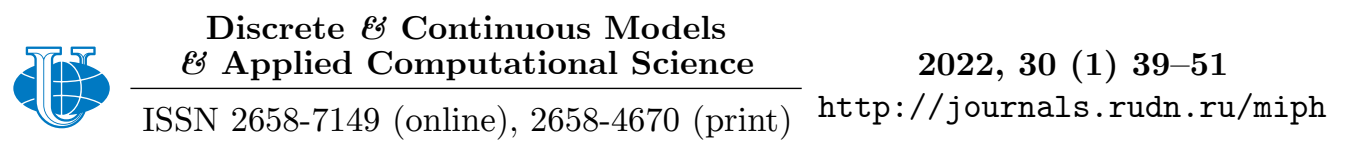

Research article

UDC 519.711.3

DOI: $10.22363 / 2658-4670-2022-30-1-39-51$

\title{
The quantization of the classical two-dimensional Hamiltonian systems
}

\author{
Irina N. Belyaeva \\ Belgorod State National Research University \\ 85, Pobedy St., Belgorod, 308015, Russian Federation
}

(received: December 26, 2021; revised: January 18, 2022; accepted: February 18, 2022)

\begin{abstract}
The paper considers the class of Hamiltonian systems with two degrees of freedom. Based on the classical normal form, according to the rules of Born-Jordan and Weyl-MacCoy, its quantum analogs are constructed for which the eigenvalue problem is solved and approximate formulas for the energy spectrum are found. For particular values of the parameters of quantum normal forms using these formulas, numerical calculations of the lower energy levels were performed, and the obtained results were compared with the known data of other authors. It was found that the best and good agreement with the known results is obtained using the Weyl-MacCoy quantization rule. The procedure for normalizing the classical Hamilton function is an extremely time-consuming task, since it involves hundreds and even thousands of polynomials for the necessary transformations. Therefore, in the work, normalization is performed using the REDUCE computer algebra system. It is shown that the use of the Weyl-MacCoy and Born-Jordan correspondence rules leads to almost the same values for the energy spectrum, while their proximity increases for large quantities of quantum numbers, that is, for highly excited states. The canonical transformation is used in the work, the quantum analog of which allows us to construct eigenfunctions for the quantum normal form and thus obtain analytical formulas for the energy spectra of different Hamiltonian systems. So, it is shown that quantization of classical Hamiltonian systems, including those admitting the classical mode of motion, using the method of normal forms gives a very accurate prediction of energy levels.
\end{abstract}

Key words and phrases: Hamilton function, normal form, Weyl-MacCoy rules, Born-Jordan rule, quantum normal form, computer modeling, energy spectra

\section{Introduction}

Representation of the original classical Hamilton function in normal form as the sum of homogeneous polynomials in canonically conjugate coordinates and momenta [1] allows us to carry out its quantum-mechanical description.

The main provisions of the new quantum mechanics were discovered by W. Heisenberg in 1925 [2]. In the same year, the paper was published by

(C) Belyaeva I. N., 2022

This work is licensed under a Creative Commons Attribution 4.0 International License http://creativecommons.org/licenses/by/4.0/ 
M. Born and P. Jordan [3], in which the commutation relation for the quantummechanical operators of coordinate $\hat{q}$ and momentum $\hat{p}$ was obtained for the first time in the form

$$
\hat{p} \hat{q}-\hat{q} \hat{p}=h / 2 \pi i,
$$

as well as the rule of matching to the classical monom of the form $q^{m} p^{n}$, $(m, n=1,2,3, \ldots)$ of its quantum analog, which we present as

$$
\mathrm{BJ}\left\{q^{m} p^{n}=p^{n} q^{m}\right\}=\frac{1}{n+1} \sum_{k=0}^{n} \hat{p}^{n-k} \hat{q}^{m} \hat{p}^{k} .
$$

The results obtained by P.A.M. Dirac [4] should be added to this. In this paper P.A.M. Dirac wrote: "In a recent work [2], Heisenberg put forward a new theory, which suggests that not the equations of classical mechanics are erroneous in any way, but that mathematical operations, by which physical results are derived from these equations, need modification. Thus, all the information provided by the classical theory can be used in the new theory... We make the basic assumption that the difference of the Heisenberg products of two quantum quantities is equal to the Poisson bracket of these quantities multiplied by $i h / 2 \pi "$ :

$$
\{q, p\} \rightarrow \frac{1}{i \hbar}[\hat{q}, \hat{p}]=\frac{1}{i \hbar}(\widehat{q} \widehat{p}-\hat{p} \hat{q})=1,
$$

here $\{q ; p\}$ is the Poisson bracket, $[\hat{q}, \hat{p}]$ is the commutator for the operators, $\hat{q}, \hat{p}, \hbar$ is Planck's constant.

In 1927, G. Weyl published a paper [5], (see also [6]), in which the author, on the basis of group-theoretic ideas, proposed the following rule of correspondence between classical quantities and their quantum analogs in integral form. Let the classical function $f(q, p)$ be determined by the following Fourier integral

$$
f(q, p)=\iint \exp (i \sigma p+i \tau q) \zeta(\sigma, \tau) d \sigma d \tau
$$

then the corresponding function $F(\hat{q}, \hat{p})$ in quantum mechanics is given by

$$
F(\hat{q}, \hat{p})=\iint \exp (i \sigma \hat{p}+i \tau \hat{q}) \zeta(\sigma, \tau) d \sigma d \tau
$$

and the operators $\hat{q}, \hat{p}$ satisfy the commutation relation (1). Based on these assumptions for functions of polynomial form $f(q, p)=q^{m} p^{n}$, a number of different relations were obtained [7], one of which can be written as

$$
\mathrm{WMc}\left\{q^{m} p^{n}=p^{n} q^{m}\right\}=\frac{1}{2^{n}} \sum_{k=0}^{n} \frac{n !}{k !(n-k) !} \hat{p}^{n-k} \hat{q}^{m} \hat{p}^{k},
$$

which we will call the Weyl-MacCoy quantization rule. 
In [8], the correspondence rule was obtained in the following form

$$
f(q) p^{n} \rightarrow \frac{1}{2^{n}} \sum_{k=0}^{n} \frac{n !}{k !(n-k) !} \hat{p}^{k} f(\hat{q}) \hat{p}^{n-k},
$$

which is represented by repeating anti-commutators $[\hat{a}, \hat{b}]_{+}=\hat{a} \hat{b}+\hat{b} \hat{a}$ as follows:

$$
f(q) p^{n} \rightarrow\left[\left[\ldots[f(\hat{q}), \hat{p}]_{+}, \hat{p}\right]_{+}, \ldots\right]_{+} .
$$

In addition to the main works mentioned above, there are publications in which the problem of the correspondence of classical quantities and their quantum analogs is discussed from different perspectives (see, for example, [9]-[14]).

A critical review of various quantization rules for classical Hamilton functions was carried out in [15].

In this paper, for the Hamiltonian, in general, non-integrable system with two degrees of freedom, we have received the classical normal BirkhoffGustavson form for which the corresponding quantum analogs are obtained by the Born-Jordan and Weyl-MacCoy quantization rules. For these quantum analogs, i.e., Schrödinger operators, approximate formulas for energy spectra are found. According to these formulas, for some specific numerical values of the parameters, the energy spectra were calculated and compared with the literature results obtained by direct numerical calculations.

\section{A quantum analog of the classical normal form}

The paper considers a classical system with two degrees of freedom, whose Hamilton function is

$$
\begin{gathered}
H=\frac{1}{2}\left(p_{1}^{2}+p_{2}^{2}\right)+V\left(q_{1}, q_{2}\right), \\
V\left(q_{1}, q_{2}\right)=\frac{1}{2}\left(q_{1}^{2}+q_{2}^{2}\right)+b\left(q_{1}^{2} q_{2}+\frac{1}{3} q_{2}^{3}\right)+c q_{1}^{2} q_{2}^{2}+d\left(q_{1}^{2}+q_{2}^{2}\right)^{2},
\end{gathered}
$$

where the coordinates $q_{1}, q_{2}$ and momenta $p_{1}, p_{2}$ are canonically conjugate variables, $b, c, d$ are dimensionless parameters.

Since system (5) is resonant with a frequency ratio of 1:1, when we bring it to normal form for the initial Hamilton function, we apply the canonical transformation with a valence equal to an imaginary unit [16]:

$$
\begin{gathered}
q_{1}=\frac{1}{2 i}\left(-Q_{1}+Q_{2}+P_{1}-P_{2}\right), \quad q_{2}=\frac{1}{2}\left(Q_{1}+Q_{2}+P_{1}+P_{2}\right), \\
p_{1}=\frac{1}{2}\left(Q_{1}-Q_{2}+P_{1}-P_{2}\right), \quad p_{2}=\frac{1}{2 i}\left(Q_{1}+Q_{2}-P_{1}-P_{2}\right),
\end{gathered}
$$

and its inverse transformation is written in the form 


$$
\begin{aligned}
Q_{1} & =\frac{1}{2}\left(q_{2}-i p_{2}\right)+\frac{i}{2}\left(q_{1}-i p_{1}\right), & Q_{2} & =\frac{1}{2}\left(q_{2}-i p_{2}\right)-\frac{i}{2}\left(q_{1}-i p_{1}\right), \\
P_{1} & =\frac{1}{2}\left(q_{2}+i p_{2}\right)-\frac{i}{2}\left(q_{1}+i p_{1}\right), & P_{2} & =\frac{1}{2}\left(q_{2}+i p_{2}\right)+\frac{i}{2}\left(q_{1}+i p_{1}\right) .
\end{aligned}
$$

It directly follows from expressions (7) that the variables $Q_{1}, Q_{2}$ are complex conjugate to the variables $P_{1}, P_{2}$ respectively.

Canonical transformations (7) using standard substitution

$$
p_{\nu} \rightarrow \hat{p}_{\nu}=-i \frac{\partial}{\partial q_{\nu}}, \quad q_{\nu} \rightarrow \hat{q}_{\nu}=q_{\nu}, \quad \nu=1,2
$$

with a known commutation rule (Planck constant $\hbar=1$ )

$$
\left[\hat{p}_{\nu}, \hat{q}_{\nu}\right]=i \delta_{\mu \nu}, \quad \mu, \nu=1,2
$$

$\left(\delta_{\mu \nu}\right.$ - Kronecker symbol) will be presented by us in the operator form

$$
\hat{Q}_{1}=\hat{a}_{2}^{+}+i \hat{a}_{1}^{+}, \quad \hat{Q}_{2}=\hat{a}_{2}^{+}-i \hat{a}_{1}^{+}, \quad \hat{P}_{1}=\hat{a}_{2}-i \hat{a}_{1}, \quad \hat{P}_{2}=\hat{a}_{2}+i \hat{a}_{1},
$$

where

$$
\begin{array}{ll}
\hat{a}_{1}^{+}=\frac{1}{2}\left(\hat{q}_{1}-i \hat{p}_{1}\right), & \hat{a}_{2}^{+}=\frac{1}{2}\left(\hat{q}_{2}-i \hat{p}_{2}\right), \\
\hat{a}_{1}=\frac{1}{2}\left(\hat{q}_{1}+i \hat{p}_{1}\right), & \hat{a}_{2}=\frac{1}{2}\left(\hat{q}_{2}+i \hat{p}_{2}\right),
\end{array}
$$

where the upper "+" symbol denotes Hermitian conjugation. Taking into account expressions (8), it is easy to verify that the operators (10) commute by the rule

$$
\left[\hat{a}_{\mu}, \hat{a}_{\nu}^{+}\right]=\frac{i}{2} \delta_{\mu \nu}
$$

and the operators (9) obey the rule

$$
\left[\hat{P}_{\mu}, \hat{Q}_{\nu}^{+}\right]=\delta_{\mu \nu} .
$$

However, the commutation (11) can be directly obtained from the Dirac quantization condition (3), given that the classical canonical transformation (6) has a valence equal to an imaginary unit.

From the expressions (9), (10) it follows that the operators $\hat{P}_{\nu}$ and $\hat{Q}_{\nu}$, $(\nu=1,2)$ are the annihilation and birth operators, respectively.

Using the quantization rules (2) and (4), we obtain two expressions of its quantum analogs $\hat{K}_{6}^{\mathrm{BJ}}$ and $\hat{K}_{6}^{\mathrm{WMc}}$, respectively, and each expression can be represented as the sum of the diagonal and nondiagonal parts

$$
\begin{gathered}
\hat{K}_{6}^{\mathrm{BJ}}=\hat{K}_{\text {diag }}^{\mathrm{BJ}}+\hat{K}_{\text {nondiag }}^{\mathrm{BJ}} \cdot \\
\hat{K}_{6}^{\mathrm{WMc}}=\hat{K}_{\text {diag }}^{\mathrm{WMc}}+\hat{K}_{\text {nondiag }}^{\mathrm{WMc}} .
\end{gathered}
$$


Below we present the diagonal parts only:

$$
\begin{aligned}
& \hat{K}_{\text {diag }}^{\mathrm{BJ}}=\hat{Q}_{1} \hat{P}_{1}+\hat{Q}_{2} \hat{P}_{2}+1+K_{41}\left[\left(\hat{Q}_{1} \hat{P}_{1}+\hat{Q}_{2} \hat{P}_{2}+1\right)^{2}+\frac{5}{2}\right]+ \\
& +K_{42}\left(\hat{Q}_{1} \hat{P}_{1}+\hat{Q}_{2} \hat{P}_{2}+2 \hat{Q}_{1} \hat{P}_{1} \hat{Q}_{2} \hat{P}_{2}+\frac{1}{2}\right)+K_{43}\left[\left(\hat{Q}_{1} \hat{P}_{1}-\hat{Q}_{2} \hat{P}_{2}\right)^{2}+\frac{5}{2}\right]+ \\
& +K_{61}\left[\left(\hat{Q}_{1} \hat{P}_{1}+\hat{Q}_{2} \hat{P}_{2}+1\right)^{3}+\frac{1}{4}\left(\hat{Q}_{1} \hat{P}_{1}\right)^{2}+\right. \\
& \left.+\frac{1}{4}\left(\hat{Q}_{2} \hat{P}_{2}\right)^{2}+\frac{27}{4}\left(\hat{Q}_{1} \hat{P}_{1}+\hat{Q}_{2} \hat{P}_{2}\right)+\frac{13}{2}\right]- \\
& -K_{64}\left[\left(\hat{Q}_{1} \hat{P}_{1}+\hat{Q}_{2} \hat{P}_{2}+1\right)\left(\hat{Q}_{1} \hat{P}_{1}+\hat{Q}_{2} \hat{P}_{2}+2 \hat{Q}_{1} \hat{P}_{1} \hat{Q}_{2} \hat{P}_{2}+3\right)\right]+ \\
& +K_{66}\left[\left(\hat{Q}_{1} \hat{P}_{1}+\hat{Q}_{2} \hat{P}_{2}+1\right)\left(\left(\hat{Q}_{1} \hat{P}_{1}-\hat{Q}_{2} \hat{P}_{2}\right)^{2}+\frac{1}{4}\left(\hat{Q}_{1} \hat{P}_{1}+\hat{Q}_{2} \hat{P}_{2}\right)\right)-\right. \\
& \left.-\frac{1}{2} \hat{Q}_{1} \hat{P}_{1} \hat{Q}_{2} \hat{P}_{2}\right] \text {. } \\
& \hat{K}_{\mathrm{diag}}^{\mathrm{WMc}}=\hat{Q}_{1} \hat{P}_{1}+\hat{Q}_{2} \hat{P}_{2}+1+K_{41}\left[\left(\hat{Q}_{1} \hat{P}_{1}+\hat{Q}_{2} \hat{P}_{2}+1\right)^{2}+\frac{1}{2}\right]+ \\
& +K_{42}\left(\hat{Q}_{1} \hat{P}_{1}+\hat{Q}_{2} \hat{P}_{2}+2 \hat{Q}_{1} \hat{P}_{1} \hat{Q}_{2} \hat{P}_{2}+\frac{1}{2}\right)+K_{43}\left[\left(\hat{Q}_{1} \hat{P}_{1}-\hat{Q}_{2} \hat{P}_{2}\right)^{2}+\frac{1}{2}\right]+ \\
& +K_{61}\left[\left(\hat{Q}_{1} \hat{P}_{1}+\hat{Q}_{2} \hat{P}_{2}+1\right)^{3}+2\left(\hat{Q}_{1} \hat{P}_{1}+\hat{Q}_{2} \hat{P}_{2}+1\right)\right]- \\
& -K_{64}\left[\left(\hat{Q}_{1} \hat{P}_{1}+\hat{Q}_{2} \hat{P}_{2}+1\right)\left(\hat{Q}_{1} \hat{P}_{1}+\hat{Q}_{2} \hat{P}_{2}+2 \hat{Q}_{1} \hat{P}_{1} \hat{Q}_{2} \hat{P}_{2}+1\right)\right]+ \\
& +K_{66}\left[\left(\hat{Q}_{1} \hat{P}_{1}+\hat{Q}_{2} \hat{P}_{2}+1\right)\left(\left(\hat{Q}_{1} \hat{P}_{1}\right)^{2}+\left(\hat{Q}_{2} \hat{P}_{2}\right)^{2}-2 \hat{Q}_{1} \hat{P}_{1} \hat{Q}_{2} \hat{P}_{2}+1\right)\right] \text {. }
\end{aligned}
$$

We note that the quantum state vectors [2]

$$
\begin{gathered}
|N, L\rangle=\left[\left(\frac{N+L}{2}\right) !\left(\frac{N-L}{2}\right) !\right]^{-1 / 2} \hat{Q}_{2}^{\left(\frac{N-L}{2}\right)} \hat{Q}_{1}^{\left(\frac{N+L}{2}\right)}|0,0\rangle, \\
\hat{P}_{1}|0,0\rangle=\hat{P}_{2}|0,0\rangle=0,
\end{gathered}
$$

where $N$ is the main quantum number, $N=0,1,2,3, \ldots$, and $L$ is the orbital quantum number, which for a given value $N$ takes the following values: $L= \pm N, \pm(N-2), \pm(N-4), \ldots, \pm 1(0)$, are eigenvectors for the diagonal parts of quantum analogs (12) and (13). The presence of nondiagonal terms in quantum analogs (12) and (13) is due to the fact that in the original classical Hamiltonian system (5) there is a 1:1 resonance ratio between frequencies.

\section{The energy spectra of quantum normal forms}

Since vectors (14) represent an orthonormal basis, the energy spectra of quantum normal forms (12) and (13) are determined by the following expressions: 


$$
\begin{aligned}
E_{\mathrm{BJ}}^{N L} & =\left\langle N, L\left|\hat{K}_{\mathrm{diag}}^{\mathrm{BJ}}\right| N, L\right\rangle+\sum_{N^{\prime}, L^{\prime}}\left\langle N^{\prime}, L^{\prime}\left|\hat{K}_{\text {nondiag }}^{\mathrm{BJ}}\right| N, L\right\rangle, \\
E_{\mathrm{WMc}}^{N L} & =\left\langle N, L\left|\hat{K}_{\text {diag }}^{\mathrm{WMc}}\right| N, L\right\rangle+\sum_{N^{\prime}, L^{\prime}}\left\langle N^{\prime}, L^{\prime}\left|\hat{K}_{\text {nondiag }}^{\mathrm{WMc}}\right| N, L\right\rangle .
\end{aligned}
$$

Using the relations

$$
\hat{Q}_{1} \hat{P}_{1}|N, L\rangle=\left(\frac{N+L}{2}\right)|N, L\rangle, \quad \hat{Q}_{2} \hat{P}_{2}|N, L\rangle=\left(\frac{N-L}{2}\right)|N, L\rangle,
$$

from expressions (15) and (16) without taking into account the nondiagonal terms, we obtain the formulas for the energy spectra

$$
\begin{aligned}
& E_{N L}^{\mathrm{BJ}}=N+1+\frac{1}{2} K_{41}\left(2 N^{2}+4 N+7\right)+\frac{1}{2} K_{42}\left(N^{2}+2 N-L^{2}+1\right)+ \\
& \frac{1}{2} K_{43}\left(2 L^{2}+5\right)+\frac{1}{8} K_{61}\left(8 N^{3}+25 N^{2}+78 N+L^{2}+60\right)- \\
& \frac{1}{2} K_{64}\left(N^{3}+3 N^{2}+8 N-N L^{2}-L^{2}+3\right)+ \\
& +\frac{1}{8} K_{66}\left(N^{2}+14 N+8 N L^{2}+9 L^{2}+12\right), \\
& E_{N L}^{\mathrm{WMc}}=N+1+\frac{1}{2} K_{41}\left(2 N^{2}+4 N+3\right)+\frac{1}{2} K_{42}\left(N^{2}+2 N-L^{2}+1\right)+ \\
& +\frac{1}{2} K_{43}\left(2 L^{2}+1\right)+K_{61}\left(N^{3}+3 N^{2}+5 N+3\right)- \\
& -\frac{1}{2} K_{64}\left(N^{3}+3 N^{2}+2 N-N L^{2}-L^{2}+1\right)+ \\
& +K_{66}\left(N+N L^{2}+L^{2}+1\right) \text {. }
\end{aligned}
$$

As it can be seen, the energy spectrum in both cases of quantization is degenerate by sign of the orbital quantum moment $L$. Besides, taking into account the contributions of nondiagonal terms can lead to a shift of energy levels, which differ in the value of the orbital quantum number by four and six units. Therefore, it is expected that approximate formulas (17), (18) with satisfactory accuracy describe the energy spectrum of the lowest states in the vicinity of a stationary point located at the origin.

From the comparison of formulas (17), (18) for energy spectra, a general conclusion can be drawn that the quantization rules of Born-Jordan and Weyl-MacCoy predict different values for the ground state energy, which are determined by the numerical values of the parameters $b, c$ and $d$.

More specific conclusions can be obtained by comparing the results of numerical calculations using formulas (17), (18) with exact energy levels calculated for any particular values of the parameters of the Hamiltonian, which will be performed in the next section. In cases where the classical 
system (5) is integrable, approximate formulas (17), (18), expressed directly through the parameters $b, c$ and $d$ have the following form.

1. If there is a relationship $c=4 d$, and the parameter $b$ is not equal to or is equal to zero, then the energy spectra are calculated by the formulas:

$$
\begin{aligned}
& E_{N L}^{\mathrm{BJ}}=N+1+d\left(\frac{9}{4} N^{2}+\frac{9}{2} N-\frac{3}{4} L^{2}+6\right)- \\
&-d^{2}\left(\frac{85}{8} N^{3}+\right.\left.\frac{1037}{32} N^{2}+\frac{1479}{16} N-\frac{187}{32} L^{2}-\frac{51}{8} N L^{2}+\frac{561}{8}\right)- \\
&-b^{2}\left(\frac{5}{8} N^{2}+\frac{5}{4} N-\frac{5}{24} L^{2}+\frac{5}{3}\right)+ \\
&+ d b^{2}\left(\frac{125}{8} N^{3}+\frac{1525}{32} N^{2}+\frac{2175}{16} N-\frac{275}{32} L^{2}-\frac{75}{8} N L^{2}+\frac{825}{8}\right)- \\
&-b^{4}\left(\frac{1175}{864} N^{3}+\right.\left.\frac{14335}{3456} N^{2}+\frac{6815}{576} N-\frac{2585}{3456} L^{2}-\frac{235}{288} N L^{2}+\frac{2585}{288}\right) \\
& E_{N L}^{\mathrm{WMc}}=N+1+d\left(\frac{9}{4} N^{2}+\frac{9}{2} N-\frac{3}{4} L^{2}+3\right)+ \\
&+d^{2}\left(\frac{17}{8} N^{3}+\frac{51}{8} N^{2}+\frac{17}{4} N-\frac{51}{8} L^{2}-\frac{51}{8} N L^{2}\right)- \\
& \quad-b^{2}\left(\frac{5}{8} N^{2}+\frac{5}{4} N-\frac{5}{24} L^{2}+\frac{5}{6}\right)- \\
&-d b^{2}\left(\frac{25}{8} N^{3}+\frac{75}{8} N^{2}+\frac{25}{4} N-\frac{75}{8} L^{2}-\frac{75}{8} N L^{2}\right)+ \\
&+b^{4}\left(\frac{235}{864} N^{3}+\frac{235}{288} N^{2}+\frac{235}{432} N-\frac{235}{288} L^{2}-\frac{235}{288} N L^{2}\right)
\end{aligned}
$$

2. If the parameters $b=c=0$, but the parameter $d>0$, then the formulas have the form:

$$
\begin{aligned}
E_{N L}^{\mathrm{BJ}}=N+1+ & d\left(\frac{3}{2} N^{2}+3 N-\frac{1}{2} L^{2}+4\right)- \\
& -d^{2}\left(\frac{17}{4} N^{3}+13 N^{2}+\frac{75}{2} N-2 L^{2}-\frac{9}{4} N L^{2}+\frac{57}{2}\right), \\
E_{N L}^{\mathrm{WMc}}=N+1+ & d\left(\frac{3}{2} N^{2}+3 N-\frac{1}{2} L^{2}+2\right)- \\
& -d^{2}\left(\frac{17}{4} N^{3}+\frac{51}{4} N^{2}+19 N-\frac{9}{4} L^{2}-\frac{9}{4} N L^{2}+\frac{21}{2}\right) .
\end{aligned}
$$

3. If the parameters $b=0, c=-2 d, d \neq 0$, then we obtain the formulas:

$$
E_{N L}^{\mathrm{BJ}}=N+1+d\left(\frac{9}{8} N^{2}+\frac{9}{4} N-\frac{3}{8} L^{2}+3\right)-
$$




$$
\begin{aligned}
& -d^{2}\left(\frac{85}{32} N^{3}+\frac{1037}{128} N^{2}+\frac{1479}{64} N-\frac{187}{128} L^{2}-\frac{51}{32} N L^{2}+\frac{561}{32}\right), \\
E_{N L}^{\mathrm{WMc}} & =N+1+d\left(\frac{9}{8} N^{2}+\frac{9}{4} N-\frac{3}{8} L^{2}+\frac{3}{2}\right)- \\
& -d^{2}\left(\frac{187}{32} N^{3}+\frac{561}{32} N^{2}+\frac{391}{16} N-\frac{153}{32} L^{2}-\frac{153}{32} N L^{2}+\frac{51}{4}\right) .
\end{aligned}
$$

\section{The comparison of energy spectra}

Unfortunately, the exact spectrum obtained, for example, by direct numerical calculations of the Schrödinger equation with its quantum analog of the original Hamilton function (5), in which a well-known replacement is to be made $p_{1} \rightarrow \hat{p}_{1}=-i \frac{\partial}{\partial q_{1}}, p_{2} \rightarrow \hat{p}_{2}=-i \frac{\partial}{\partial q_{2}}, q_{1} \rightarrow \hat{q}_{1}=q_{1}, q_{2} \rightarrow \hat{q}_{2}=q_{2}$ for arbitrary values of its parameters, is not available in the literature.

Also, direct numerical calculations using modern computer technologies face the difficulty of solving eigenvalue problems, for example, even with the help of carefully developed software packages based on the diagonalization method, which is also the task of integrating the Schrödinger equation for two or more independent variables.

Below we present the results of numerical calculations of energy spectra for specific numerical values of the parameters $b, c, d$ in cases where the classical system (5) is integrable.

Table 1 shows the values of the lowest energy levels calculated by the approximate formulas (19) and (20) in the first case of integrability, i.e., under the condition $c=4 d$ and $b=0$.

Table 1

The comparison of energy levels at parameter values $b=0, c=0.02, d=0.005,(c=4 d)$

\begin{tabular}{|l|l|l|l|l|l|l|}
\hline No & $E_{N, L}$ & $E_{N L}^{\mathrm{BJ}}$ & $E_{N L}^{\mathrm{WMc}}$ & $E_{N L}^{\mathrm{BJ}}-E_{N L}^{\mathrm{WMc}}$ & $E_{n+1}^{\mathrm{WMc}}-E_{n}^{\mathrm{WMc}}$ & $E_{n+1}^{\mathrm{BJ}}-E_{n}^{\mathrm{BJ}}$ \\
\hline 1. & $E_{0,0}$ & 1.028247 & 1.015000 & 0.013247 & - & - \\
2. & $E_{1, \pm 1}$ & 2.055166 & 2.045000 & 0.010166 & 1.030000 & 1.026919 \\
3. & $E_{2, \pm 2}$ & 3.095512 & 3.089363 & 0.006149 & 1.044363 & 1.039953 \\
4. & $E_{2,0}$ & 3.108259 & 3.106275 & 0.001984 & 0.016913 & 0.013141 \\
5. & $E_{3, \pm 3}$ & 4.147469 & 4.147450 & 0.000019 & 1.041175 & 1.039209 \\
6. & $E_{3, \pm 1}$ & 4.172475 & 4.182550 & -0.010075 & 0.035100 & 0.025006 \\
7. & $E_{4, \pm 4}$ & 5.211578 & 5.218625 & -0.007047 & 1.036075 & 1.039103 \\
8. & $E_{4, \pm 2}$ & 5.247175 & 5.273188 & -0.026013 & 0.054563 & 0.035597 \\
9. & $E_{4,0}$ & 5.259041 & 5.291375 & -0.032334 & 0.018188 & 0.011866 \\
\hline
\end{tabular}


From the table 1, it follows that the Weyl-MacCoy quantization rule leads to a lower energy level for the ground state and a greater decomposition of the levels with respect to the orbital moment at a given value of the principal quantum number $N$. In the classically integrable case under consideration, there are no exact (analytical or numerical) values of the energy spectrum in the current literature. However, the spectrum is known [17] in the second classical case of integrability, when the parameters of the quantum analog of the Hamilton function (5) are equal $b=0, c=0, d \neq 0$.

Besides, the values of the energy spectrum of a one-dimensional anharmonic oscillator are known and also with great accuracy, in particular, with a fourth degree in potential energy. Knowing this spectrum, it is possible to construct an approximate spectrum of a quantum analog of the original Hamilton function (5), but already a two-dimensional Hamiltonian given the values of parameters $b=0, c=-2 d, d \neq 0$, for which system (5) is integrable in the classical case.

We will compare below these well-known and very reliable numerical results for the energy spectra with our results, which are calculated by formulas (23), (24) according to the Born-Jordan and Weil-McCoy quantization rules.

For parameter values $b=0, c=0$ and $d \neq 0$ the Schrödinger equation corresponding to the classical Hamilton function (5) allows separation of variables in polar coordinates, and the energy spectrum is characterized by a radial quantum number $n$ and orbital momentum $l$. In [17] a method for numerical solving the radial Schrödinger equation was developed and energy levels were calculated for the values of quantum numbers equal $n, l=0,1,2$ for a parameter value $d=0.000005$. Quantum numbers $n, l$ are connected with our numbers $N, L$ by the following relations: $N=2 n+l,|L|=l$.

Table 2 shows the energy levels obtained in [17], as well as their values calculated for the same value of the parameter using formulas (21) and (22) based on quantization of the classical normal form, according to the Born-Jordan and Weil-McCoy rules, respectively.

Table 2 shows that a very good approximation to the exact spectrum is given by the application of the Weyl-MacCoy quantization rule. In particular, the ground state energy obtained using the Weyl-MacCoy quantization rule differs from the result of [17] by $0.5 \cdot 10^{-7 \%}$, and when quantized by the Born-Jordan rule, by $0.001 \%$. At the same time, for energy of level 14, these errors are equal, respectively, $0.4 \cdot 10^{-8 \%}$ and $0.0001 \%$, i.e., the prediction according to the Born-Jordan rule improves.

In the third case $(b=0, c=-2 d, d \neq 0)$ of integrability of the classical system (5), with its quantum-mechanical description, it is necessary to solve the following two-dimensional Schrödinger equation

$$
\left(\hat{H}_{1}+\hat{H}_{2}\right) \Psi=2 E \Psi, \quad \hat{H}_{i}=-\frac{d^{2}}{d q_{i}^{2}}+q_{i}^{2}+2 d q_{i}^{4}, \quad i=1,2,
$$

where the variables are separated. Therefore, its solving is reduced to solving two identical one-dimensional equations for the anharmonic oscillator, and the energy spectrum is found in the form of the following sum $2 E=2 E_{1}+2 E_{2}$.

The quantum numbers of an isotropic two-dimensional oscillator $(N, L)$ are connected with the quantum numbers $(n 1, n 2)$ of one-dimensional oscillators 
by the following relations: $N=n 1+n 2$ and $L=n 1-n 2$. We note that the ordering of the values of the energy spectrum levels by the value of quantum numbers $(N, L)$ as compared to another numbering of states has the advantage that the values of the energy spectrum levels, numbered by quantum numbers $(N, L)$, grow with an increase of the main quantum number.

Table 2

The comparison of energy levels $E_{N L}^{\mathrm{BJ}}$ and $E_{N L}^{\mathrm{WMc}}$ with their values from [17] for $d=0.000005$

\begin{tabular}{|c|l|c|c|c|}
\hline No. & $2 E_{N, L}$ & $E_{N L}^{\mathrm{BJ}}$ & $E_{N L}^{\mathrm{WMc}}$ & Results [17] \\
\hline 1. & $2 E_{0,0}$ & 2.0000399985 & 2.0000199995 & 2.0000199995 \\
2. & $2 E_{1, \pm 1}$ & 4.0000799961 & 4.0000599979 & 4.0000599981 \\
3. & $2 E_{2, \pm 2}$ & 6.0001399918 & 6.0001199946 & 6.0001199949 \\
4. & $2 E_{2,0}$ & 6.0001599905 & 6.0001399933 & 6.0001399936 \\
5. & $2 E_{3, \pm 3}$ & 8.0002199853 & 8.0001999892 & 8.0001999892 \\
6. & $2 E_{3, \pm 1}$ & 8.0002599818 & 8.0002399856 & 8.0002399859 \\
7. & $2 E_{4, \pm 4}$ & 10.000319975 & 10.000299981 & 10.000299981 \\
8. & $2 E_{4, \pm 2}$ & 10.000379969 & 10.000359974 & 10.000359975 \\
9. & $2 E_{4,0}$ & 10.000399967 & 10.000379971 & 10.000379972 \\
10. & $2 E_{5, \pm 5}$ & 12.000439962 & 12.000419969 & 12.000419969 \\
11. & $2 E_{5, \pm 3}$ & 12.000519952 & 12.000499958 & 12.000499958 \\
12. & $2 E_{5, \pm 1}$ & 12.000559947 & 12.000539953 & 12.000539953 \\
13. & $2 E_{6, \pm 6}$ & 14.000579946 & 14.000559953 & 14.000559953 \\
14. & $2 E_{6, \pm 4}$ & 14.000679930 & 14.000659937 & 14.000659937 \\
15. & $2 E_{6, \pm 2}$ & 14.000739921 & 14.000719928 & 14.000719929 \\
16. & $2 E_{6,0}$ & 14.000759918 & 14.000739925 & 14.000739925 \\
\hline
\end{tabular}

\section{Conclusions}

In this paper for a classical system with two degrees of freedom with the Hamilton function (5), a classical normal form is obtained in the BirkhoffGustavson approach, for which its quantum analogs are constructed according to the Born-Jordan and Weyl-MacCoy heuristic quantization rules. For these quantum analogs, which are nothing but approximate differential expressions for the exact Schrödinger operator, the eigenvalue problem is solved and the formulas of energy spectra are found. 
Using these formulas, in two special cases with specific numerical values of the parameters, the lower energy levels were calculated and the results obtained were compared with the data available in the works published by other authors. It was found that the best and good agreement with the known results of calculating the energy spectrum is obtained using the Weyl-MacCoy quantization rule in comparison with the Born-Jordan rule.

Both the Weyl-MacCoy and Born-Jordan quantization rules are derived from the fundamental, but different postulates of classical and quantum mechanics. For the system under consideration, particular numerical results for the energy spectrum reveal the advantage of the Weyl-MacCoy quantization rule, however, it is probably premature to extend this conclusion to other systems.

\section{References}

[1] N. N. Chekanova, I. K. Kirichenko, V. E. Bogachev, and N. A. Chekanov, "The classical and quantum approach in the study of a nonlinear Hamiltonian system," Bulletin of the Tambov State University. Series "Natural and Technical Sciences", vol. 20, no. 1, pp. 120-137, 2015.

[2] W. Heisenberg, "Über quanten theoretische Umdeutung kinematischer und mechanischer Beziehungen," Zeitschrift für Physik, vol. 33, pp. 879893, 1925. DOI: $10.1007 / \mathrm{BF} 01328377$.

[3] M. Born and P. Jordan, "Zur quanten mechanik," Zeitschrift für Physik, vol. 34, pp. 858-888, 1925. DOI: 10.1007/BF01328531.

[4] P. Digas, "Fundamental Equations of Quantum Mechanics," Proc. Roy Soc. (Lnd.)., pp. 642-653, 1925. DOI: 10.3367/UFNr.0122.197708e. 0611.

[5] H. Weyl, "Quanten Mechanik und Gruppen Theorie," Zeitschrift für Physik, vol. 46, pp. 1-46, 1927. DOI: 10.1063/1.1664478.

[6] G. Weil, The theory of groups and quantum mechanics. Martino Fine Books, 2014.

[7] N. H. McCoy, "On the function in quantum mechanics which corresponds to a given function in classical mechanics," Proceedings of the National Academy of Sciences (PNAS), vol. 18, pp. 674-676, 1932. DOI: 10.1073/ pnas.18.11.674.

[8] S. R. De Groot and L. G. Suttorp, Foundations of Electrodynamics. Amsterdam: North-Holland publishing company, 1972. DOI: 10.12691/ amp-2-3-6.

[9] A. N. Argyers, "The Bohr-Sommerfeld quantization rule and the Weyl correspondence," Physics, vol. 2, p. 131, 1965. DOI: 10.1103/ PhysicsPhysiqueFizika.2.131.

[10] L. Castellani, "Quantization rules and Dirac's correspondence," Il Nuovo Cimento A, vol. 48, pp. 359-368, 1978. DOI: 10.1007/BF02781602. 
[11] P. Crehan, "The parametrisation of quantization rules equivalent to operator orderings and the effect of different rules on the physical spectrum," Journal of Physics A: Mathematical and General, vol. 22, no. 7, pp. 811-822, 1989. DOI: 10.1088/0305-4470/22/7/013.

[12] P. Crehan, "The proper quantum analogue of the Birkhoff-Gustavson method of normal forms," Journal of Physics A: Mathematical and General, vol. 23, no. 24, pp. 5815-5828, 1990. DOI: 10.1088/03054470/23/24/022.

[13] W. A. Fedak and J. J. Prentis, "The 1925 Born and Jordan paper "On quantum mechanics"," American Journal of Physics, vol. 77, pp. 128 139, 2009. DOI: 10.1119/1.3009634.

[14] M. A. Gosson, "Born-Jordan quantization and the uncertainty principle," Journal of Physics A: Mathematical and Theoretical, vol. 46, pp. 445462, 2013. DOI: 10.1088/1751-8113/46/44/445301.

[15] M. Razavy, Heisenberg's quantum mechanics. Singapore: World Scientific Publishing Co. Pte. Ltd., 2011. DOI: 10.1080/00107514.2011.603435.

[16] N. A. Chekanov, "Quantization of the normal form of BirkhoffGustavson [Kvantovaniye normal'noy formy Birkgofa-Gustavsona]," Nuclear Physics, vol. 50, no. 8, pp. 344-346, 1989, in Russian.

[17] H. Taseli, "On the Exact Solution of the Schroedinger Equation with a Quartic Anharmonicity," International Journal of Quantum Chemistry, vol. 57, no. 1, pp. 63-71, 1996. DOI: 10.1002/(SICI) 1097-461X (1996) $57: 1<63:$ :AID-QUA7>3.0.CO;2-X.

\section{For citation:}

I. N. Belyaeva, The quantization of the classical two-dimensional Hamiltonian systems, Discrete and Continuous Models and Applied Computational Science 30 (1) (2022) 39-51. DOI: 10.22363/2658-4670-2022-30-1-39-51.

\section{Information about the authors:}

Belyaeva, Irina N. - Candidate of Physical and Mathematical Sciences, Associate Professor of Belgorod State National Research University (e-mail: ibelyaeva@bsu.edu.ru, ORCID: https://orcid.org/0000-0002-1368) 
УДК 519.711 .3

DOI: $10.22363 / 2658-4670-2022-30-1-39-51$

\title{
Квантование классических двумерных гамильтоновых систем
}

\author{
И. Н. Беляева \\ Белгородский государственный исследовательский университет \\ ул. Победъ, д. 85, Белгород, 308015, Россия
}

\begin{abstract}
Аннотация. В статье рассматривается класс гамильтоновых систем с двумя степенями свободы. На основе классической нормальной формы, согласно правилам Борна-Йордана и Вейля-Маккоя, построены её квантовые аналоги, для которых решена задача на собственные значения и найдены приближённые формулы для энергетического спектра. Для конкретных значений параметров квантовых нормальных форм с использованием этих формул были проведены численные расчёты нижних энергетических уровней, полученные результаты были сопоставлены с известными данными других авторов. Обнаружено, что наилучшее согласие с известными результатами достигается с использованием правила квантования Вейля-Маккоя. Процедура нормализации классической функции Гамильтона является крайне трудоёмкой задачей, так как вовлекает сотни и даже тысячи многочленов для необходимых преобразований. Поэтому в работе нормализация выполняется с помощью системы компьютерной алгебры REDUCE. Показано, что использование правил соответствия БорнаЙордана и Вейля-Маккоя приводит практически к одним и тем же значениям для энергетического спектра, при этом их близость увеличивается для больших величин квантовых чисел, то есть для высоковозбуждённых состояний. В работе использовано каноническое преобразование, квантовый аналог которого позволяет построить собственные функции для квантовой нормальной формы и получить таким образом аналитические формулы для энергетических спектров разных гамильтоновых систем. Итак, показано, что квантование классических гамильтоновых систем, в том числе допускающих классический режим движения, с применением метода нормальных форм даёт очень точное предсказание уровней энергии.
\end{abstract}

Ключевые слова: функция Гамильтона, нормальная форма, правило ВейляМаккоя, правило Борна-Йордана, квантовая нормальная форма, компьютерное моделирование, энергетические спектры 